\title{
NF-KB p65-dependent transcriptional regulation of histone deacetylase 2 contributes to the chronic constriction injury-induced neuropathic pain via the microRNA-183/TXNIP/NLRP3 axis
}

\author{
Jiamin Miao ${ }^{1 *}$, Xuelong Zhou' ${ }^{2}$ Tianjiao $\mathrm{Ji}^{3}$ and Gang Chen ${ }^{1 *}$
}

\begin{abstract}
Background: Neuropathic pain is related to the sustained activation of neuroglial cells and the production of proinflammatory cytokines in the spinal dorsal horn. However, the clinical efficacy of currently available treatments is very limited. The transcription factor nuclear factor $\mathrm{KB}(\mathrm{NF}-\mathrm{KB})$ is a ubiquitously expressed protein family and considered to be crucial in autoimmunity. Thus, our study aimed to examine the influence of NF-kB p65 in chronic constriction injury (CCl)-induced neuropathic pain as well as its underlying mechanism.
\end{abstract}

Methods: A rat model of neuropathic pain was established by $\mathrm{CCl}$ induction followed by isolation of microglial cells. The binding of NF-KB p65 to HDAC2, of miR-183 to TXNIP, and of TXNIP to NLRP3 was investigated. Expression of miR-183, NF-KB p65, HDAC2, TXNIP, and NLRP3 was determined with their functions in CCI rats and microglial cells analyzed by gain- and loss-of-function experiments.

Results: NF-KB p65 and HDAC2 were upregulated while miR-183 was downregulated in the dorsal horn of the CCl rat spinal cord. NF-KB p65 was bound to the HDAC2 promoter and then increased its expression. HDAC2 reduced miR-183 expression by deacetylation of histone H4. Additionally, miR-183 negatively regulated TXNIP. Mechanistically, NF-KB p65 downregulated the miR-183 expression via the upregulation of HDAC2 and further induced inflammatory response by activating the TXNIP-NLRP3 inflammasome axis, thus aggravating the neuropathic pain in $\mathrm{CCl}$ rats and microglial cells.

Conclusion: These results revealed a novel transcriptional mechanism of interplay between NF-KB and HDAC2 focusing on neuropathic pain via the miR-183/TXNIP/NLRP3 axis.

Keywords: Neuropathic pain, Chronic constriction injury, NF-KB p65, Histone deacetylase 2, microRNA-183, TXNIP, NLRP3

\footnotetext{
*Correspondence: jmmiao@zju.edu.cn; chengang120@zju.edu.cn

'Department of Anesthesiology, Sir Run Run Shaw Hospital, Zhejiang

University School of Medicine, No. 3, Qingchun East Road, Jianggan District, Hangzhou 310012, Zhejiang Province, China

Full list of author information is available at the end of the article
}

C C The Author(s). 2020 Open Access This article is licensed under a Creative Commons Attribution 4.0 International License, which permits use, sharing, adaptation, distribution and reproduction in any medium or format, as long as you give appropriate credit to the original author(s) and the source, provide a link to the Creative Commons licence, and indicate if changes were made. The images or other third party material in this article are included in the article's Creative Commons. licence, unless indicated otherwise in a credit line to the material. If material is not included in the article's Creative Commons licence and your intended use is not permitted by statutory regulation or exceeds the permitted use, you will need to obtain permission directly from the copyright holder. To view a copy of this licence, visit http://creativecommons.org/licenses/by/4.0/. The Creative Commons Public Domain Dedication waiver (http://creativecommons.org/publicdomain/zero/1.0/) applies to the data made available in this article, unless otherwise stated in a credit line to the data. 


\section{Background}

Neuropathic pain is a universal disease in clinical practice, with the prevalence ranging from 7 to $10 \%$ in the general population [1]. The most common causes of neuropathic pain include inflammation-induced central and peripheral neuron sensitization in the spinal dorsal horn [2]. Patients with neuropathic pain exhibit frequent and severe depression, sleep disturbances, and anxiety, which further enhance the burden on their life quality [3]. However, due to the complex pathogenesis of neuropathic pain, effective therapy remains a great challenge [4]. Chronic constriction injury (CCI) is a widely used model to induce neuropathic pain in experimental animals [5]. Thus, it is urgently required to explore the molecular mechanism and develop innovative targets to relieve $\mathrm{CCI}$-induced neuropathic pain.

Accumulating data have demonstrated that nuclear factor-kappa B (NF-kB), which transfers receptormediated signals to the nucleus to regulate the pro- and antinociceptive factors, is implicated in the process of neuropathic pain [6]. Accordingly, Yin et al. have elucidated that NF- $\mathrm{kB}$ inhibitor (pyrrolidine dithiocarbamate, PDTC) plays a suppressive role in neuropathic pain [7]. Moreover, NF- $\mathrm{kB}$ is regarded as a crucial regulator of histone deacetylase 2 (HDAC2) [8], while histone deacetylase 2 (HDAC2), a member of HDAC, is reported to be involved in neuropathic pain [9]. Importantly, HDAC2 exhibits significant effects on the progression of dorsal horn following peripheral injury [10]. A previous study has revealed that the depletion of HDAC could regulate the microRNA-183 (miR-183) to affect the tumor process [11]. Also, previously reported studies have verified the impact of miR-183 in the regulation of CCI-induced neuropathic pain [12]. Nevertheless, our results from microarray analysis further verified that miR-183 could affect the neuropathic pain by regulating the thioredoxin interacting protein (TXNIP)/NLR family pyrin domain-containing 3 (NLRP3) inflammasome axis in peripheral nerve injury. TXNIP (also known as thioredoxin binding protein-2 or vitamin D3 upregulated binding protein2 ) is considered as a key regulator for the formation of the NLRP3 inflammasome [13], whilst the elevation of NLRP3 inflammasome has been proved to aggravate the process of neuropathic pain [14]. Yet, the specific mechanisms of NF- $\mathrm{kB}$ p65-mediating HDAC2 to regulate the miR-183/TXNIP/NLRP3 axis in neuropathic pain remain poorly understood. Thus, in the present study, CCI models were established to explore the role of NF- $\mathrm{kB}$ p65-mediating HDAC2 inflammatory response in neuropathic pain through the miR183/TXNIP/NLRP3 axis along with its possible mechanisms, which may help to provide a novel direction for treating neuropathic pain.

\section{Materials and methods}

\section{Ethics statement}

Animal experiments were approved by the Ethics Committees of Zhejiang University School of Medicine and conducted by following the Guide for the Care and Use of Laboratory Animals published by the US National Institutes of Health. All efforts were made to minimize the number and suffering of the included animals.

\section{$\mathrm{CCl}$ rat model establishment}

Sprague-Dawley (SD) male rats weighing 180-200 g (purchased from SLAC Laboratory Animal Co. Ltd. Shanghai, China) were used in this study. After adaptive feeding for 1 week, the rats were given a normal diet and drinking water, after which they were randomly divided into the normal control group, sham group, and CCI group (10 rats in each group). The CCI rat model was established as previously described [2]. Briefly, the rats were anesthetized by intraperitoneal injection of $3 \%$ pentobarbital sodium at a dose of $40 \mathrm{mg} / \mathrm{kg}$, and the sciatic nerve of rat right thigh was exposed by blunt dissection. Proximal to the sciatic nerve, about $7 \mathrm{~mm}$ of the nerve without adhering tissue and four ligatures (about $1 \mathrm{~mm}$ interval) were ligated around the sciatic nerve until a brief twitch was observed. The rats in the sham group were subjected to the same procedures as mentioned before except for the ligation. The surgical incision was disinfected with iodine, and the wound was sutured.

\section{Animal treatment}

The CCI rats (14 days of CCI induction) were treated with $50 \mathrm{mg} / \mathrm{kg} \mathrm{NF-kB} \mathrm{p65} \mathrm{inhibitor} \mathrm{PDTC} \mathrm{(once} \mathrm{a} \mathrm{day}$ for 14 days via intraperitoneal injection), lentiviral vectors containing overexpression (oe)-HDAC2 (injected once via tail vein), $20 \mathrm{mg} / \mathrm{kg}$ SAHA (HDAC inhibitor, once a day for 14 days via intraperitoneal injection), lentiviral vectors containing short hairpin (sh)-HDAC2 (injected once via tail vein), miR-183 agomir (injected once via tail vein), and lentiviral vectors containing oeTXNIP (injected once via tail vein). The scrambled shRNA vector and agomir negative control (NC) were served as NCs. NF-kB p65 inhibitor PDTC (Beyotime Institute of Biotechnology, Shanghai, China) and SAHA inhibitor (Sigma-Aldrich Chemical Company, St Louis, MO, USA) were dissolved in the 5\% dimethyl sulfoxide (DMSO), respectively, sub-packed, and preserved at -20 ${ }^{\circ} \mathrm{C}$. After 14 days, the rats were euthanized after anesthesia using $5 \%$ pentobarbital sodium $(30 \mathrm{mg} / \mathrm{kg})$ (P3761, Sigma-Aldrich Chemical Company, St Louis, MO, USA). The dorsal horn of the spinal cord of rats was isolated and collected for detecting the expression of related genes. 


\section{Cell culture and microglial cell isolation}

Human embryo kidney (HEK293T) cells (American Type Culture Collection [ATCC], Manassas, VA, USA, CRL-1573) were grown in Dulbecco's modified Eagle's medium (DMEM) containing 10\% fetal bovine serum (FBS), and cultured in a $5 \% \mathrm{CO}_{2}$ incubator at $37^{\circ} \mathrm{C}$. Microglial cells were isolated from the dorsal horn of the spinal cord in SD rats and cultured according to the previously reported method [15]. Microglial cells were added with DMEM/F12 (Gibco, Carlsbad, California, USA) containing 10\% FBS (Hyclone Laboratories, Logan, UT, USA), $100 \mathrm{U} / \mathrm{mL}$ penicillin, and $100 \mathrm{mg} / \mathrm{mL}$ streptomycin and then cultured in a flask $\left(75 \mathrm{~cm}^{2}\right)$ for 14 days. At day 10, microglial cells were isolated from primary mixed glial cell culture by shaking on a rotary oscillator at $300 \mathrm{rpm}$ at $37^{\circ} \mathrm{C}$ overnight. After 2 days, the CD11b antibody (\#31-1174-00, BD Biosciences, Franklin Lakes, NJ, USA) was adopted to measure the purity of primary microglial cells, which was over $95 \%$ of purity.

\section{Construction and packaging of lentiviral vectors}

The lentivirus packaging system was constructed via LV5-GFP (lentivirus gene overexpression vector) and pSIH1-H1-copGFP (lentivirus gene silencing vector). HDAC2 shRNA, NF-kB p65 shRNA, miR-183 mimic, mimic NC, and scramble shRNA were provided by Shanghai GenePharma Co. Ltd. (Shanghai, China). The packaging lentivirus and target vector were cotransfected into the HEK293T cells and cultured for 48 $h$. The supernatant was then collected, and the lentivirus particles in the supernatant were filtered to detect virus titer. Then, the lentiviruses in the exponential phase were collected, and the cells were infected with lentiviruses containing sh-NC, sh-HDAC2, oe-NC, oe-TXNIP, mimic NC, miR-183 mimic, and miR-183 mimic + oeTXNIP, respectively. When the cells grew to the logarithmic growth phase, they were trypsinized and mechanically dissociated into cell suspension $\left(5 \times 10^{4}\right.$ cells/ $\mathrm{mL})$ which was then seeded into 6-well plates $(2 \mathrm{~mL} /$ well) and incubated at $37^{\circ} \mathrm{C}$ overnight. After $48 \mathrm{~h}$ of transfection, reverse transcription-quantitative polymerase chain reaction (RT-qPCR) was used to detect the expression of related genes in cells.

\section{Intrathecal catheters and drug treatment}

For spinal drug administration, all male SD rats were implanted with intrathecal catheters by inserting them into the subarachnoid space between the L5 and L6 vertebrae. After surgical procedures, the rats were housed individually and had free access to water and food which was provided. On the 2 nd day after surgical procedure, the rats were injected with $2 \%$ lidocaine $(30 \mu \mathrm{L})$ through catheters over $30 \mathrm{~s}$ followed by a $10-\mu \mathrm{L}$ flush of normal saline. Hind paw paralysis and/or paresis within $30 \mathrm{~s}$ and lasting 6-10 min indicated a successful catheterization. Rats exhibiting postoperative neurologic deficits (about $25 \%)$ were excluded. The shRNA corresponding to NF$\kappa B$ p65, HDAC, TXNIP, and NC was cloned into a pFUGW-RNAi-GFP vector (Shanghai Gene Chem Co., Ltd., Shanghai, China). To determine the level of infection efficiency of lentivirus constructs, LV-GFP was preliminarily studied. Then, the lentivirus was injected into the rats, and the experiments were conducted four weeks later.

\section{Neuropathic pain behavior test}

Mechanical allodynia and thermal hyperalgesia were measured to assess the response of rats to neuropathic pain [2]. Behavior test was carried out at 1 day before the surgical procedure at days $1,3,5,7$, and 14 , respectively, after sciatic nerve injury in the rat right thigh. Rats were placed individually in a wire mesh cage and habituated for 30-60 min. Calibrated von Frey filaments in the mesh cage were applied to the plantar surface of the rat hind paw from below the mesh floor. Thermal punctuate stimuli were transferred to the plantar surface of the hind paw with a focused beam of radiant heat, and the withdrawal latency time was recorded. The $50 \%$ mechanical paw withdrawal threshold (PWT) was determined using the up-down method. Thermal hyperalgesia was assessed by measuring the latency of paw withdrawal in response to a radiant heat source. Thermal sensitivity was determined by the thermal paw withdrawal latency (PWL). The result of each test was expressed as the mean of three withdrawal latencies in seconds. Rats were acclimated to the test environment at least $30 \mathrm{~min}$ before the commencement of the experiments. Behavioral analysis was performed between 09: 00 and 12:00 in a quiet room.

\section{Dual-luciferase reporter assay}

The 3'untranslated region (3'UTR) of TXNIP binding with miR-183 was inserted into the dual-luciferase reporter gene vector pmirGLO. Site-directed mutagenesis of wild-type (WT) reporter gene plasmid pmirGLOTXNIP-WT was carried out using a mutation kit. Then, the reporter plasmids were co-transfected with miR-183 mimic and NC mimic (both were purchased from Shanghai GenePharma Co. Ltd., Shanghai, China) into $293 \mathrm{~T}$ cells. After transfection for $24 \mathrm{~h}$, the cells were lysed at room temperature for $15 \mathrm{~min}$ to obtain the supernatant. The luciferase activity was measured using a dual-Luciferase Reporter Assay System (E1910, Promega, Madison, WI, USA). Thereafter, $20 \mu \mathrm{L}$ samples were added with $100 \mu \mathrm{L}$ firefly luciferase and $100 \mu \mathrm{L}$ Renilla luciferase working solution to detect the activity of firefly luciferase and Renilla luciferase, respectively. The ratio of firefly luciferase activity to internal Renilla 
luciferase activity was regarded as the relative luciferase activity. Each sample was repeated more than 3 times.

\section{Western blot analysis}

Following 14 days of CCI construction, the tissue samples of the dorsal horn of rat spinal cord were washed with pre-cooled phosphate-buffered saline (PBS), lysed with radioimmunoprecipitation assay (RIPA) lysis buffer (C0481, Sigma-Aldrich Chemical Company, St Louis, MO, USA) on ice for $30 \mathrm{~min}$, and centrifuged at $12000 \mathrm{~g}$ and $4{ }^{\circ} \mathrm{C}$ for $15 \mathrm{~min}$. The supernatant was then collected, and the protein concentration was measured using the bicinchoninic acid (BCA) protein concentration test kit (Beyotime). A total of $20 \mu \mathrm{g}$ protein samples were subjected to separation by $10 \%$ sodium dodecyl sulfatepolyacrylamide gel electrophoresis (SDS-PAGE) and transferred onto a polyvinylidene fluoride (PVDF) membrane (Millipore, Billerica, MA, USA) at a constant current of $250 \mathrm{~mA}$. The membranes were blocked with $5 \%$ skimmed milk powder at ambient temperature for 1 $\mathrm{h}$ followed by overnight incubation at $4{ }^{\circ} \mathrm{C}$ with primary rabbit antibodies to NF- $\mathrm{kB}$ phosphorylated (p)-p65 (\#3037, 1:500, Cell Signaling Technology), NF-кB P65 (ab16502, 1:1000, Abcam, Cambridge, UK), HDAC2 (ab16032, 1: 500, Abcam), TXNIP (ab188865, 1:1000, Abcam), NLRP3 (ab232401, 1: 1000, Abcam), apoptosisassociated Speck-like protein containing a CARD (ASC; ab47092, 1: 1000, Abcam), caspase-1 (ab179515, 1: 1000, Abcam), and glyceraldehyde-3-phosphate dehydrogenase (GAPDH; ab181602, 1: 1000, Abcam). The next day, the membrane was incubated with horseradish peroxidaselabeled secondary antibody to immunoglobulin G (IgG) (ab99702, 1:1000, Abcam) for $1 \mathrm{~h}$ at room temperature. Thereafter, the membrane was developed using enhanced chemiluminescence (Shanghai Baoman Biotechnology Co., Ltd., Shanghai, China), and the gray value was analyzed using the Image $J$ software. The protein level was represented by the ratio of the gray value of target bands to that of the internal reference (GAPDH).

\section{RNA isolation and quantitation}

The expression of NF-kB p65, HDAC2, TXNIP, NLRP3, and miR-183 in the dorsal horn of rat spinal cord and microglial cells was determined using the RT-qPCR. Dorsal horn of spinal cord and microglial cells was lysed using the TRIzol reagent (Invitrogen, Carlsbad, California, USA) followed by total RNA extraction with phenol-chloroform at room temperature for 10-30 min. The purity $(260 / 280=1.8-2.0)$ and concentration of the extracted RNA were then determined by a nucleic acid quantitative instrument. Subsequently, $400 \mathrm{ng}$ of the extracted RNA was transcribed into complementary DNA (cDNA) using the PrimeScript RT Reagent Kit (Takara Bio Inc., Otsu, Shiga, Japan). RT-qPCR was conducted using the chimeric dye $\mathrm{SYBR}^{\bullet}$ Premix Ex $\mathrm{Taq}^{\mathrm{TM}}$ II kit (Takara Bio Inc., Otsu, Shiga, Japan). Amplification was conducted using the Thermal Cycler Dice Real-Time System (TP800, Takara). The primers for RT-qPCR were synthesized by Guangzhou RiboBio Co., Ltd. (Guangzhou, China) (Table 1) while the primers for miR-183 were designed using the stem-loop method [16]. With GAPDH regarded as the internal reference of mRNAs and U6 of miRNA, the expression of the target gene was calculated using the $2^{-\Delta \Delta \mathrm{Ct}}$ method [16]. The experiment was repeated at least 3 times independently.

\section{Immunofluorescence staining}

Following 14 days of CCI model construction, the transverse spinal cord of rats was cut into $25-\mu \mathrm{m}$-thick cryostat sections. Afterward, the tissue sections were soaked twice in PBS, fixed with $4 \%$ paraformaldehyde at room temperature for $15 \mathrm{~min}$, washed twice with PBS, treated with $0.5 \%$ Triton $\mathrm{X}-100$ for $10 \mathrm{~min}$, and washed twice with PBS. Then, the sections were incubated with normal donkey serum (\#017-000-121, Jackson ImmunoResearch, West Grove, PA, USA) at room temperature for $2 \mathrm{~h}$. Subsequently, the sections were incubated with primary rabbit antibodies to NF-kB p-p65 (ab16502, 1:200, Abcam), HDAC2 (ab32117, 1:200, Abcam), TXNIP (1: 50; sc-271238, Santa Cruz Biotechnology, Santa Cruz, CA, USA), NLRP3 (ab4207, 1:100, Abcam), and goatanti Iba-1 polyclonal antibody (ab5076, 1: 500, Abcam) at $4{ }^{\circ} \mathrm{C}$ for $24 \mathrm{~h}$. The next day, the sections were reprobed with secondary antibodies to the Alexa Fluor 647 (ab15007, 1: 500, Abcam) or the Alexa Fluor 488 (ab150137, 1:500, Abcam) in the dark at $37^{\circ} \mathrm{C}$ for $2 \mathrm{~h}$. The nucleus was stained using $5 \mu \mathrm{g} / \mathrm{mL}$ DAPI at room temperature for $5 \mathrm{~min}$, and the fluorescence decayresistant medium was added for blocking. The image

\begin{tabular}{ll} 
Table 1 Primer sequences for RT-qPCR \\
\hline Target & Primer sequences (5'-3') \\
\hline NF-KB p65 & F: 5'-GCCTGACACCAGCATTTGA-3' \\
R: 5'-CAAACCAAACAGCCTCACG-3' \\
FDAC2
\end{tabular}


was observed under an inverted fluorescence microscope (FV1000, Olympus Corp., Tokyo, Japan). The same part of the fluorescence image was selected, and the Image J software was used to analyze the superimposed integrated optical density (IOD), with more than 5 samples in each group. The expression of NF-kB p-p65, HDAC2, TXNIP, and NLRP3 in Iba-1-labeled microglial cells was analyzed using a laser confocal microscopy.

\section{Enzyme-linked immunosorbent assay (ELISA)}

The tissue samples of the spinal dorsal horn of CCI rats were collected after 14 days. The tissue samples were added with $60 \mu \mathrm{L}$ RIPA cell lysis (Beyotime Institute of Biotechnology, Shanghai, China) and centrifuged at $12000 \mathrm{rpm}$ and $4{ }^{\circ} \mathrm{C}$ for $10 \mathrm{~min}$ to collect the supernatant. Then, $10 \mu \mathrm{L}$ samples were obtained to detect protein concentration using the BCA protein assay kit. The subsequent procedures were conducted according to interleukin (IL)-1 $\beta$ ELISA Kit. Then, the optical density (OD) value of each well (96-well plates) was measured at the wavelength of $562 \mathrm{~nm}$ using ELISA (Vafioskan Flash, Thermo, USA). The standard curve was drawn with protein concentration as the $\mathrm{X}$-axis and the $\mathrm{OD}$ value as the $\mathrm{Y}$-axis. According to the $\mathrm{OD}$ value of the sample well to be tested, the IL-1 $\beta$ concentration was calculated according to the standard curve and expressed as $\mathrm{pg} / \mathrm{mg}$ of protein.

\section{Co-immunoprecipitation (Co-IP) assay}

IP assay was conducted according to the instructions of Catch and Release Reversible Immunoprecipitation System Kit (Merck Millipore, Billerica, MA, USA). The antibodies used for IP assay were as follows: antibody to TXNIP-1 (sc271238, Santa Cruz Biotechnology, Santa Cruz, CA, USA) and antibody to NLRP3 (ab4207, Abcam). Rabbit/mouse IgG served as an NC for the IP reaction.

\section{Chromatin immunoprecipitation (ChIP) assay}

The ChIP assay was conducted as previously reported [16]. In short, the cultured microglial cells were washed twice with precooled PBS and added with $1 \%$ paraformaldehyde for $5 \mathrm{~min}$ of crosslinking at room temperature, which was halted with $127 \mathrm{mM}$ glycine. The cells were then lysed in the ChIP-grade cell lysis buffer containing $50 \mathrm{mM}$ Tris $\mathrm{HCl}$ (pH 8.1), 1\% SDS, 10 $\mathrm{mM}$ ethylenediaminetetraacetic acid (EDTA), and complete protease inhibitor mixture (Roche Diagnostics $\mathrm{GmbH}$, Mannheim, Germany) on ice for $30 \mathrm{~min}$. Then, the cells were subjected to ultrasonic treatment to make the DNA fragments (200-1000 bp). The subsequent ChIP assay was conducted using the ChIP Assay Kit (Millipore). Briefly, $1 \mu \mathrm{g}$ DNA was added with protein A agarose and ChIP-grade anti-ac-H4 (ab15823, Abcam) or anti-HDAC2 (sc-81599, Santa Cruz Biotechnology) for incubation overnight $4{ }^{\circ} \mathrm{C}$. Normal mouse antibody to IgG (sc-2025, Santa Cruz Biotechnology) or normal rabbit antibody to IgG (sc-2027, Santa Cruz Biotechnology) served as NC. The next day, the DNA complex was washed with low-salt and high-salt buffer and treated with protease $\mathrm{K}$ at $56^{\circ} \mathrm{C}$ for $2 \mathrm{~h}$, after which the DNA was extracted and purified by phenol/chloroform. The primers for specific ChIP-qPCR amplification of miR183 promoter region were $\mathrm{F}$ : $5^{\prime}$-CGTAGGGCCACTGG ACGA-3' and R: 5' -TTGTCCCCATTCCAGCCCTG-3'.

\section{Statistical analysis}

All data analyses were conducted using the SPSS 21.0 software (IBM Corp. Armonk, NY, USA). Measurement data conforming to the normal distribution and homogeneity of variance were expressed as the mean \pm standard deviation. Data between the two groups were compared using unpaired $t$ test while data among multiple groups were compared by one-way analysis of variance (ANOVA) followed by Tukey's post hoc test with corrections for multiple comparisons. Data comparisons at different time points were conducted using repeatedmeasures ANOVA followed by Bonferroni post hoc test. Statistical significance was set at $p<0.05$.

\section{Results}

NF-KB p65 and HDAC2 were upregulated in the dorsal horn of $\mathrm{CCl}$ rat spinal cord

CCI rat models were established and assessed by measuring the PWT and PWL. Our results exhibited the decreased PWT on the 3rd day and PWL at the 1st day after the CCI model establishment. However, a remarkable decrease in PWT and PWL was observed at the 5th, 7th, and 14th days post CCI model establishment (Fig. 1a). According to previously reported literature, NF- $\mathrm{kB}$ and HDAC play an important role in the regulation of spinal dorsal horn pain; specifically, inhibition of the inflammatory signaling, NF- $\mathrm{kB}$, could alleviate neuropathic pain [2]. The PWT and PWL of animals following CCI model construction can be increased by intrathecal injection of HDAC inhibitor [17]. Thus, in this study, we aimed to explore the expression of NF- $\mathrm{kB}$ p 65 and HDAC2 and their possible regulatory mechanisms involved in CCI rat models. Western blot analysis was first performed to determine the protein levels of NF- $\mathrm{kB}$ p 65 and HDAC2 as well as the extent of NF- $\mathrm{kB}$ p 65 phosphorylation in the dorsal horn of CCI rat spinal cord, and our results exhibited the elevated protein levels of NF- $\mathrm{B}$ p 65 and HDAC2 along with the increased extent of NF- $\mathrm{kB}$ p65 phosphorylation in dorsal horn at the 3rd, 7th, and 14th days post CCI model establishment (Fig. $1 b, c)$. Furthermore, the expression of HDAC2 and NF$\kappa B$ p-p65 in Iba-1-labeled microglial cells of the spinal 


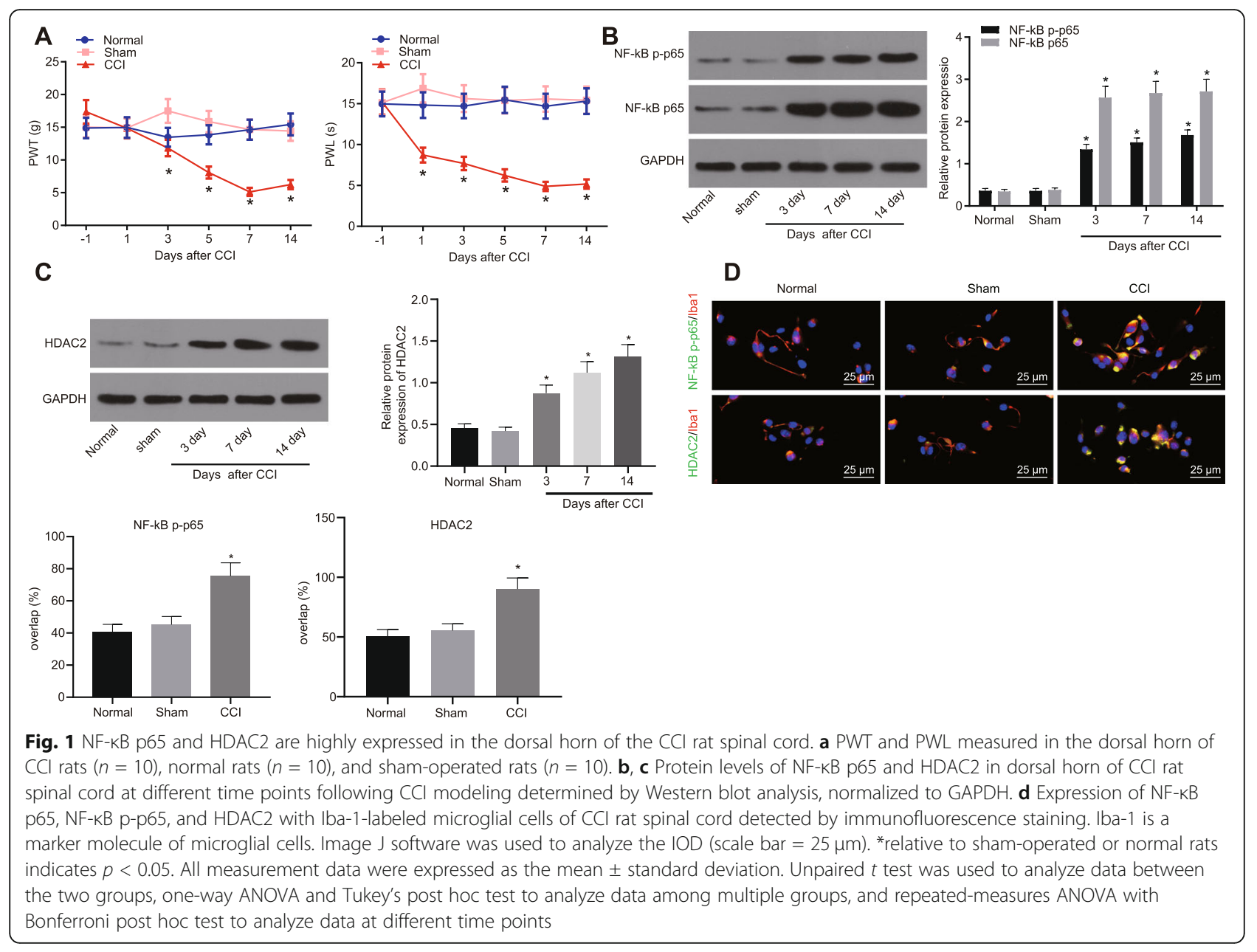

dorsal horn of CCI rats was analyzed by a laser confocal microscope followed by the analysis of the superimposed fluorescence intensity. Our findings revealed that HDAC2 and NF- $\mathrm{kB}$ p-p65 were highly expressed in microglial cells of the spinal dorsal horn in CCI rats (Fig. 1d). Thus, it can be speculated that upregulated NF-kB and HDAC2 could regulate neuropathic pain in $\mathrm{CCI}$ rats.

\section{Silencing of NF-KB p65 relieved neuropathic pain in $\mathrm{CCI}$ rats by inhibiting HDAC2 expression}

These above-described results revealed the expression pattern of NF-kB p65 and HDAC2 in CCI rats. Thereafter, we aimed to study the underlying mechanism of $\mathrm{NF}-\mathrm{kB}$ p65 regulating HDAC2 in neuropathic pain. At first, lentivirus-transduced NF- $\mathrm{kB}$ p65 knockdown plasmids were constructed, and its silencing efficiency was confirmed to be significant in microglial cells by RTqPCR and Western blot analysis (Fig. 2a). Meanwhile, treatment with PDTC resulted in a reduction of HDAC2 expression (Fig. 2b). To verify the regulatory relationship between them, we further constructed the HDAC2 promoter-reporter plasmid. The dual-luciferase reporter assay suggested that the luciferase activity of HDAC2 promoter was decreased upon NF-kB p65 knockdown (Fig. 2c). We thus hypothesized that blocking the NF-kB p65/HDAC2 signaling could alleviate the neuropathic pain in CCI rats. To further validate this finding, $\mathrm{CCI}$ rats were intraperitoneally injected with NF- $\mathrm{kB}$ p65 inhibitor PDTC $(50 \mathrm{mg} / \mathrm{kg}$, once a day, for 14 days) to analyze the effect of PDTC on PWT and PWL, our results demonstrated that PWT and PWL were significantly increased, and hyperalgesia was relieved by PDTC (Fig. 2d, e). Additionally, Western blot analysis showed that PDTC remarkably suppressed the protein expression of NF-kB p65 and HDAC2 along with reduced NF$\kappa B$ p65 phosphorylation extent in the spinal dorsal horn in CCI rats (Fig. 2f). These results suggested that NF- $\mathrm{KB}$ p65 silencing repressed the neuropathic pain in $\mathrm{CCI}$ rats by downregulating HDAC2.

\section{Silencing of HDAC2 caused miR-183 upregulation and then relieved neuropathic pain in $\mathrm{CCI}$ rats}

To further explore the molecular mechanism of HDAC2 regulating neuropathic pain in $\mathrm{CCI}$ rats, HDAC2 was 

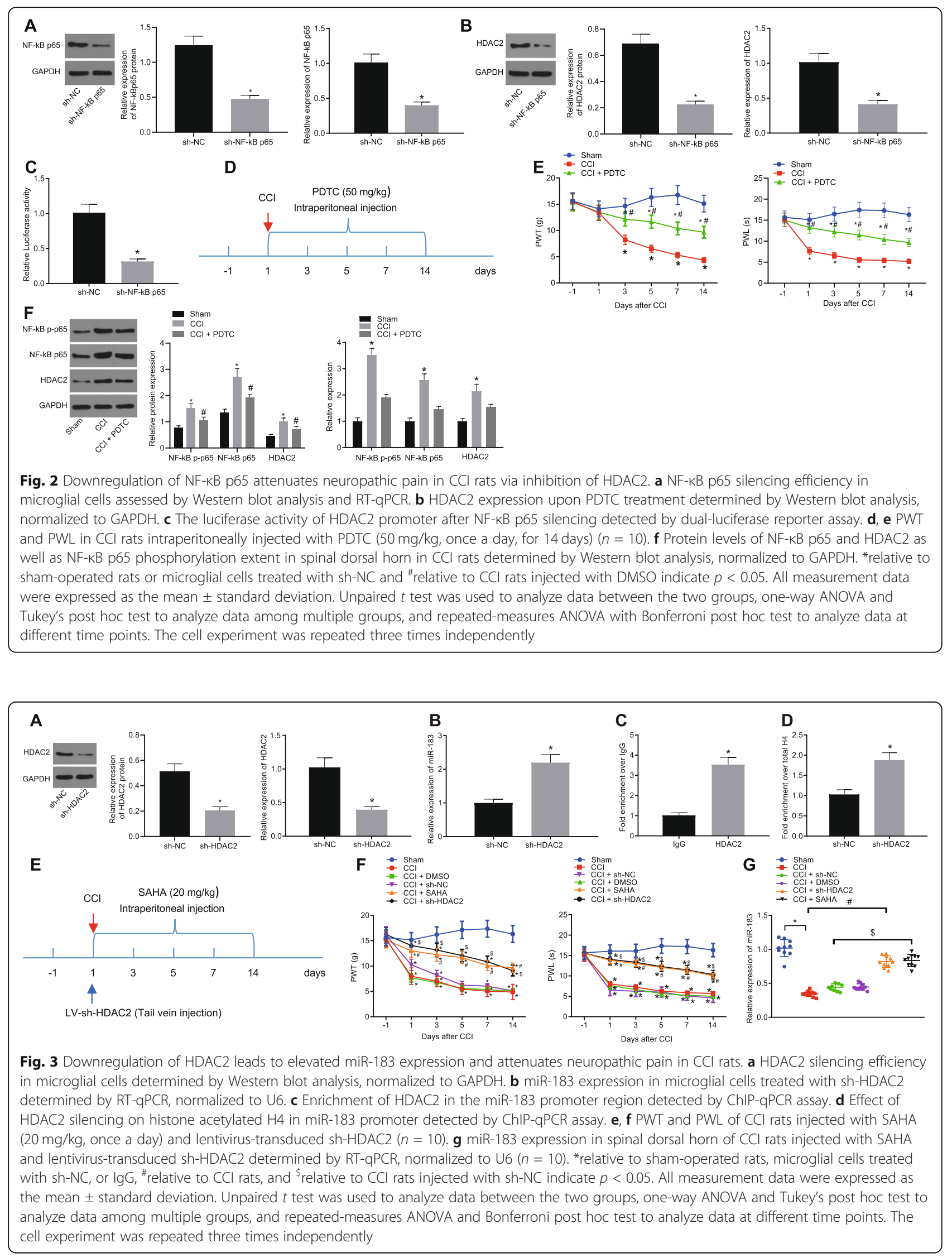
silenced in microglial cells isolated from CCI rats. Our results from Western blot depicted that lentivirustransduced sh-HDAC2 reduced the HDAC2 protein levels in microglial cells (Fig. 3a). The miR-183 expression in microglial cells was found to be increased by the lentivirus-transduced sh-HDAC2 (Fig. 3b). Moreover, the ChIP-qPCR assay described that HDAC2 was enriched in the promoter region of miR-183 (Fig. 3c), and HDAC2 silencing elevated the acetylation of histone $\mathrm{H} 4$ and then upregulated the miR-183 expression in microglial cells (Fig. 3d), suggesting that HDAC2 regulated the miR-183 transcription via $\mathrm{H} 4$ acetylation. To further confirm whether HDAC2 can mediate the regulatory mechanism of neuropathic pain in vivo, CCI rats were injected with HDAC inhibitor SAHA $(20 \mathrm{mg} / \mathrm{kg}$, once a day for 14 days) to detect the PWT and PWL. Our results depicted increased PWT and PWL after treatment with SAHA (Fig. 3e). Moreover, CCI rats were injected with lentivirus-transduced sh-HDAC2 via tail vein, and PWT and PWL were measured; the results of which revealed that the silencing of HDAC2 increased the PWT and PWL in CCI rats (Fig. $3 \mathrm{f}$ ). The results from RT-qPCR exhibited the reduced miR-183 expression in the spinal dorsal horn of CCI rats and DMSOtreated CCI rats when compared to sham-operated rats while elevated miR-183 expression was found in the spinal dorsal horn of CCI rats injected with SAHA or lentivirus-transduced sh-HDAC2 (Fig. 3g). The abovementioned evidence demonstrated that HDAC2 could downregulate the miR-183 expression while NF- $\mathrm{kB}$ p65 modulated the miR-183 transcription expression by regulating the HDAC2 in CCI rats.

\section{Upregulation of miR-183 attenuated neuropathic pain in $\mathrm{CCl}$ rats by inhibiting TXNIP expression}

Bioinformatics analysis showed binding sites between miR-183 and 3'UTR of TXNIP (Fig. 4a), which was further verified by the dual-luciferase reporter assay. As shown in Fig. 4b, elevated miR-183 could inhibit the luciferase activity of TXNIP-WT-3'UTR while it did not affect the luciferase activity of TXNIP-mutant (MUT)-3' UTR, suggesting that miR-183 could target the TXNIP. Additionally, RT-qPCR and Western blot analysis indicated that TXNIP expression was increased while miR183 expression was reduced in CCI rat models (Fig. 4c). Immunofluorescence staining revealed that TXNIP was upregulated in Iba-1-labeled microglial cells of CCI rats (Fig. 4d). Then, we further verified whether miR-183 regulates TXNIP in microglial cells. For this purpose, microglial cells were first transfected with miR-183 mimic, and RT-qPCR and Western blot analysis were performed. Our results showed that after miR-183 mimic transfection, the expression of miR-183 was significantly increased in microglial cells (Fig. 4e) while the protein level of TXNIP was significantly decreased (Fig. 4f). To further clarify the effect of miR-183 on neuropathic pain in rats, CCI rats were injected with miR-183 agomir and lentivirus-transduced oe-TXNIP via the tail vein. The response of related neuropathic pain in rats was analyzed after 14 days. The results are shown in Fig. $4 \mathrm{~g}$, $\mathrm{h}$ which revealed that miR-183 agomir triggered an increase in PWT and PWL of CCI rats, which was restored by the co-treatment with miR-183 agomir and oe-TXNIP. The RT-qPCR demonstrated that miR-183 was highly expressed but TXNIP was poorly expressed in the spinal cord of CCI rats injected with miR-183 agomir, which was negated by the dual treatment with miR-183 agomir and oe-TXNIP (Fig. 4i, j). Thus, it can be concluded that miR-183 suppressed the neuropathic pain in CCI rats by decreasing the TXNIP expression.

\section{Upregulation of miR-183 suppressed inflammatory responses in $\mathrm{CCl}$ rats by inhibiting the TXNIP-NLRP3 inflammasome axis}

Subsequently, Western blot analysis was performed to determine the expression of NLRP3 in spinal cord of CCI rats, which exhibited its upregulated expression (Fig. 5a). Our results from immunofluorescence staining revealed that NLRP3 was upregulated in microglial cells (Fig. 5b). The RT-qPCR and Western blot analysis presented that miR-183 mimic reduced the NLRP3 expression in microglial cells from CCI rats (Fig. 5c). To further determine whether TXNIP could activate NLRP3 under neuropathic pain conditions, a Co-IP assay was adopted to detect the binding of TXNIP with NLRP3. Our findings revealed that TXNIP could pull down the NLRP3 protein, whereas NLRP3 could pull down the TXNIP protein (Fig. 5d), indicating that TXNIP could activate the NLRP3 in the process of neuropathic pain. Furthermore, Western blot analysis and ELISA indicated that the protein expression of NLRP3, ASC, caspase-1, and IL- $1 \beta$ was significantly decreased in microglial cells treated with exogenous miR-183 mimic, which was rescued by treatment of oe-TXNIP (Fig. 5e, f). Western blot analysis and ELISA showed that the expression of NLRP3, ASC, caspase-1, and IL-1 $\beta$ was decreased in the spinal cord of CCI rats injected with the lentivirusexpressing miR-183 agomir, which was reversed by the overexpression of TXNIP (Fig. 5g, h). Collectively, the upregulation of miR-183 repressed inflammatory responses in CCI rats via suppression of the TXNIPNLRP3 inflammasome axis.

\section{NF-KB p65 promoted neuropathic pain in $\mathrm{CCl}$ rats by activating the miR-183-TXNIP-NLRP3 axis via HDAC2}

To further explore the mechanism of miR-183-TXNIPNLRP3 axis regulated by NF- $\mathrm{kB}$ p65 via HDAC2 in neuropathic pain in vivo, the expression of miR-183, 


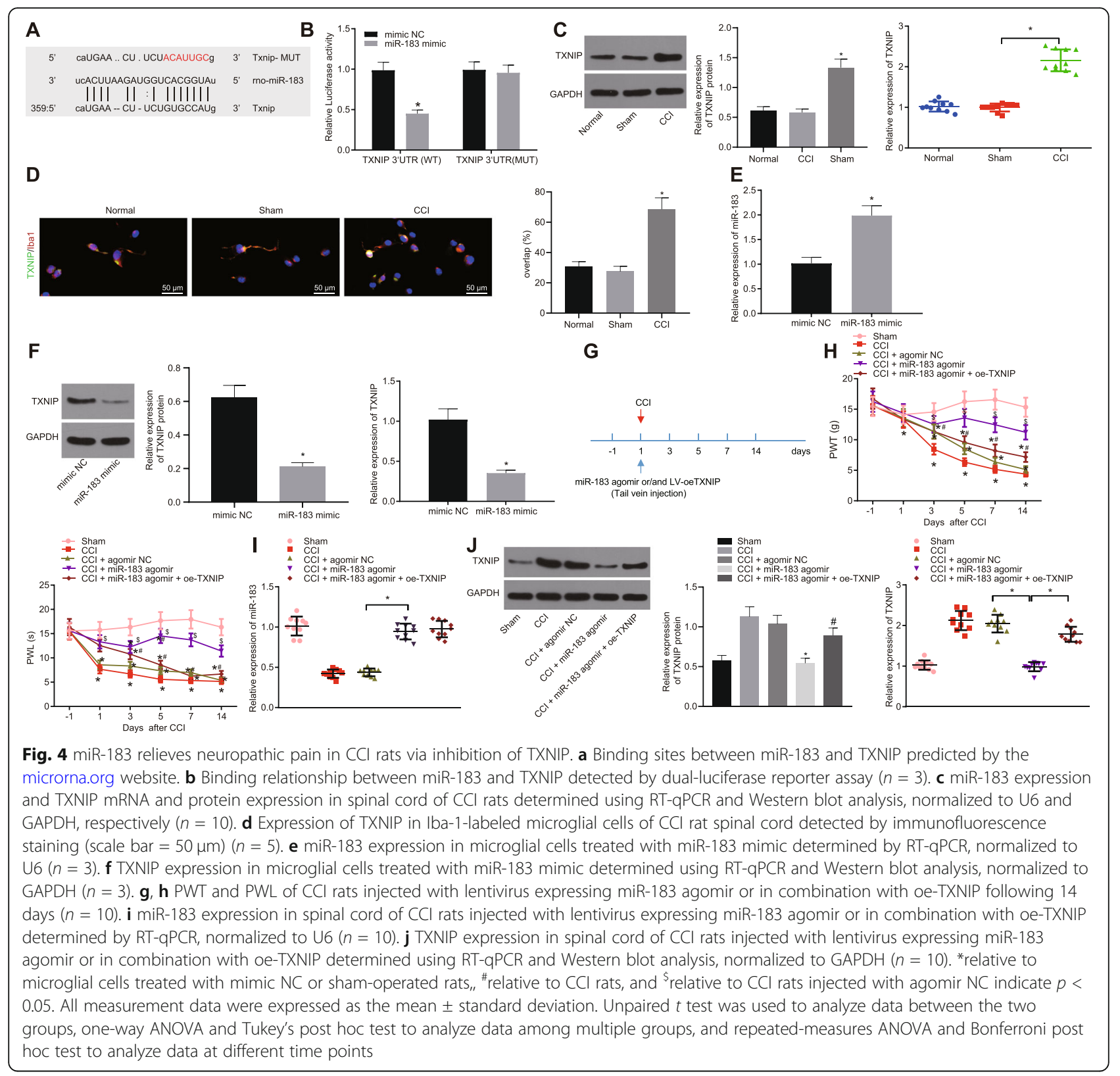

TXNIP, NLRP3, and inflammatory corpuscle-related factors, ASC, caspase- 1 , and IL- $1 \beta$ was measured in the spinal cord of CCI rats treated with NF- $\mathrm{kB}$ p65 inhibitor PDTC using the RT-qPCR and Western blot analysis. As documented in Fig. 6a-c, miR-183 expression was elevated in the spinal cord of CCI rats treated with PDTC, while a reduction was observed in the expression of TXNIP, NLRP3, ASC, caspase-1, and IL-1 $\beta$. Additionally, dual treatment with PDTC and oe-HDAC2 elevated the expression of TXNIP, NLRP3, ASC, caspase-1, and IL-1 $\beta$ in the spinal cord of CCI rats. To further explore whether this mechanism was mediated by the HDAC2, CCI rats treated with PDTC were injected with lentivirus-transduced oe-HDAC2. After 14days, the molecular level and neuropathic pain-related behavioral changes were compared in rats treated with PDTC and PDTC + oe-HDAC2 (Fig. 6d). Our results from the RTqPCR and Western blot analyses revealed that the expression of HDAC2 was higher in spinal cord of CCI rats treated with PDTC + oe-HDAC2 than in CCI rats treated with PDTC (Fig. 6e, f). Additionally, compared to PDTC-treated CCI rats, PWT and PWL were increased in CCI rats injected with PDTC + oe-HDAC2 (Fig. 6g). Taken together, NF-kB p65 could downregulate the miR-183 expression in CCI rats through HDAC2 and trigger inflammatory response via activation of the TXNIP-NLRP3 to aggravate neuropathic pain in CCI rats. 


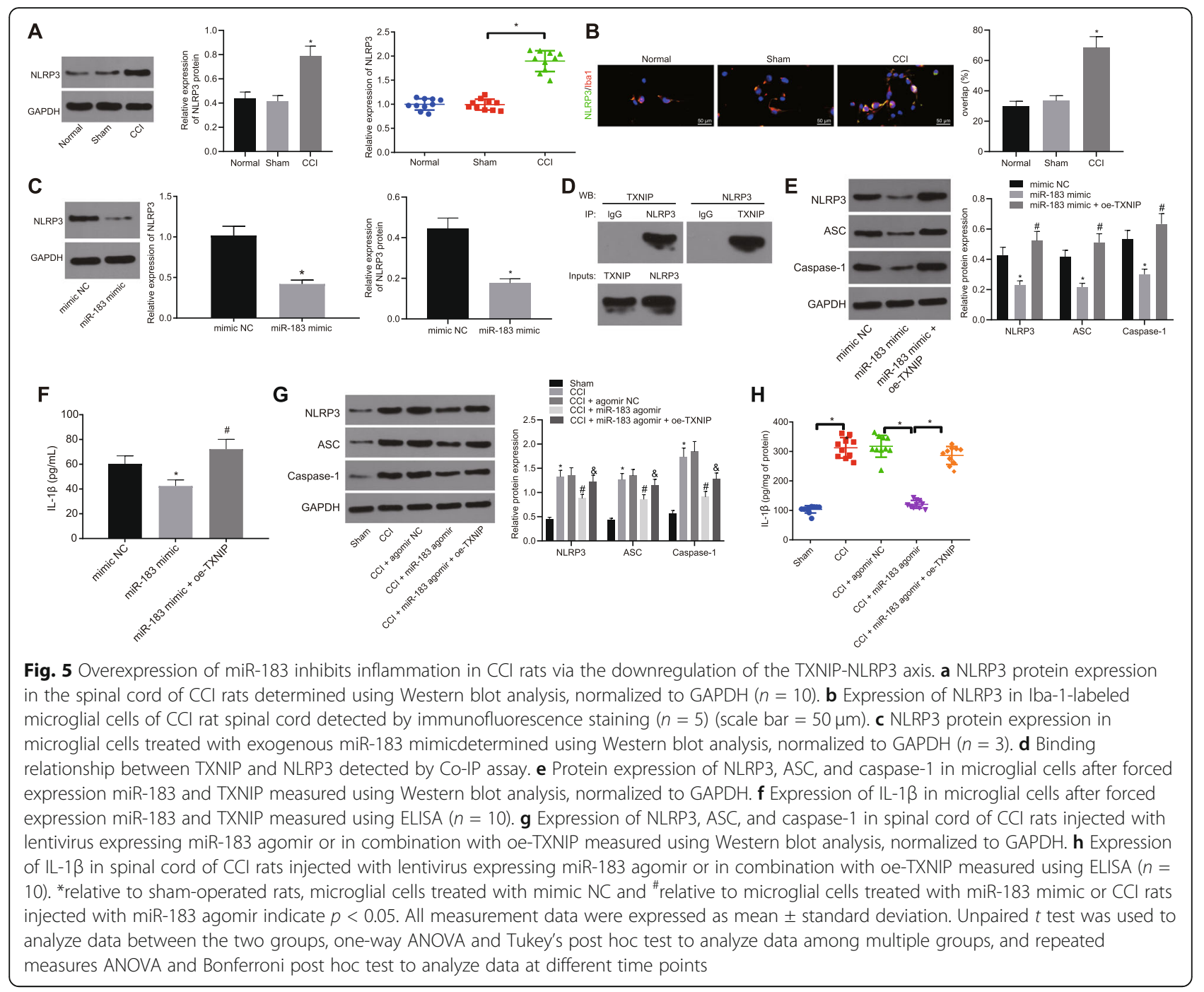

\section{Discussion}

Neuropathic pain is a frequently encountered disease that is often resistant to available treatments, exhibiting a poor prognosis in patients [1]. Previously, reported studies have demonstrated the crucial role of NF- $\mathrm{kB}$ in the progression of neuropathic pain [7]. Our study aimed to explore the encouraging effect of NF- $\mathrm{kB}$ p65mediating $\mathrm{HDAC} 2$ on inflammation in CCI-induced neuropathic pain. Taken together, our study revealed that the silencing of NF- $\mathrm{kB}$ p65 significantly reduced the expression of HDAC2, which further inhibits the inflammation and relieves neuropathic pain via the upregulation of miR-183 and downregulation of the TXNIPNLRP3 inflammasome axis.

Initially, our results demonstrated that in the dorsal horn of CCI rat spinal cord with neuropathic pain, NF$\kappa B$ p65 and HDAC2 were upregulated, whereas silencing NF- $\mathrm{KB}$ p65 suppressed the inflammatory reaction and relieved neuropathic pain in CCI rats by inhibiting the
HDAC2 expression. Accordingly, a recently reported study has verified the upregulation of NF-kB during the progression of neuropathic pain while its suppression relieved the neuropathic pain [6]. On the other hand, a well-known NF-kB inhibitor, i.e., PDTC has been reported to possess the potential to repress thermal hyperalgesia and mechanical allodynia in CCI rats [2]. Nevertheless, it is well documented that the main characteristic of neuropathic pain is the activation of inflammatory cytokines, such as IL-1 $\beta$, which suggests that targeting the inflammatory response could be a crucial factor in treating neuropathic pain [2]. NF-kb (known as a pleiotropic transcriptional factor) exhibits significant impacts in regulating the expression of multiple proinflammatory factors such as IL-6, which leads to neuropathic pain [18], whilst inhibition of NF- $\mathrm{kB}$ has been proved to attenuate pain and inhibit inflammation after peripheral nerve injury [19]. The p65 subunit of NF-kB has been reported to combine with HDAC2 as part of 


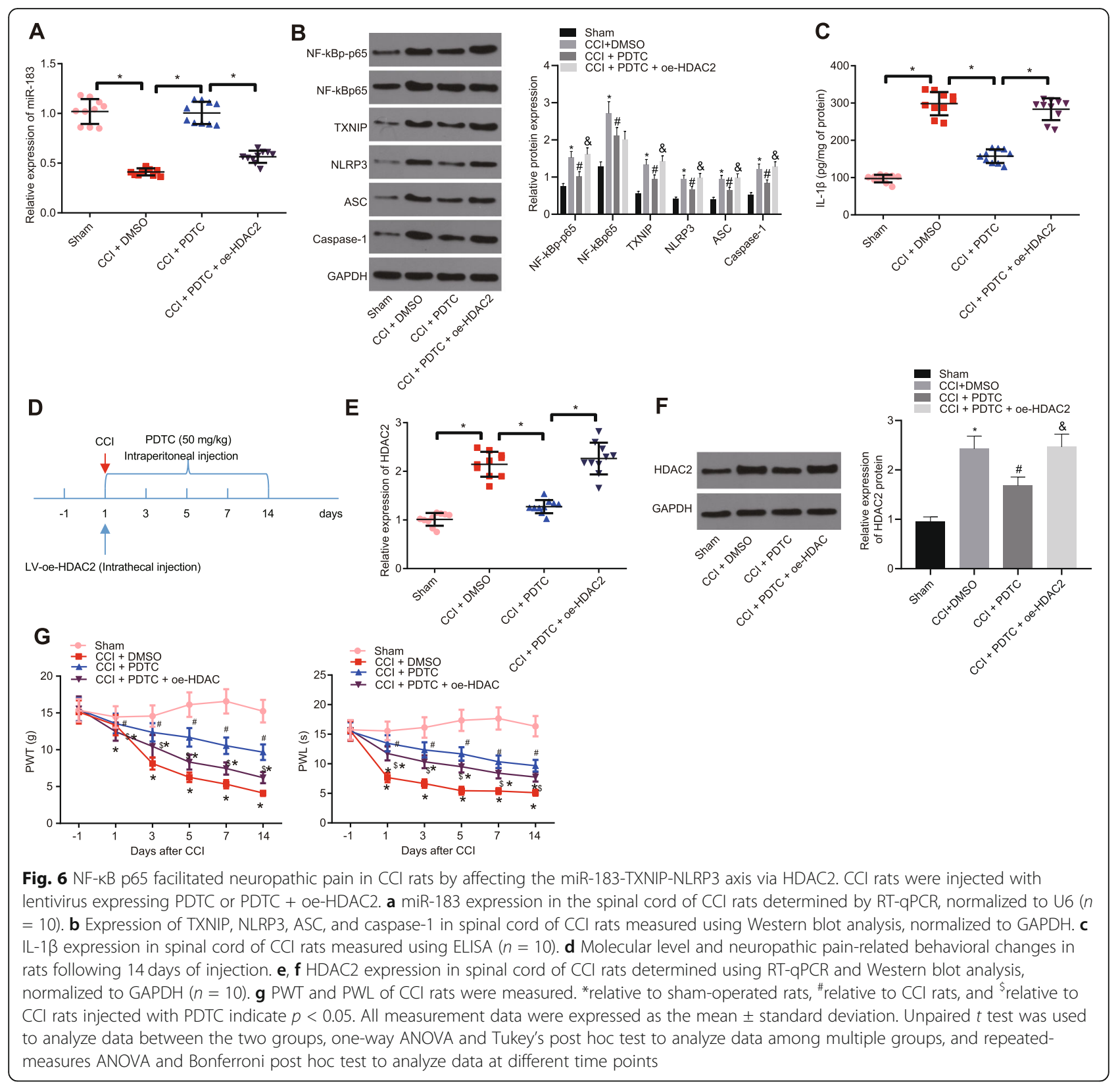

the same protein complex to negatively regulate the gene transcription in the mouse frontal cortex [20]. Thus, HDAC2 expression is positively related to NF$\kappa \mathrm{B}$ expression [8]. In consent with our findings, HDAC2 expression has been documented to elevate in the superficial dorsal horn of rats [10]. Accordingly, accumulating studies have confirmed the effects of HDAC on the molecular mechanism of neuropathic pain [17]. Importantly, HDAC2 inhibitors have been suggested to ameliorate neuropathic pain by restoring C-fibre sensitivity [21]. Hence, these abovedescribed findings supported the fact that NF-kB p65 knockdown could be considered as a potential therapeutic target to relieve neuropathic pain by suppressing HDAC2.

Furthermore, the data in the current study implied that the depletion of HDAC2 upregulated the miR-183 expression. Consistently, it has been revealed that inhibition of HDAC2 upregulated the miR-183 and exhibits tumor suppressive functions [11]. Moreover, our results from bioinformatics analysis and dual-luciferase reporter gene assay further verified that miR-183 could target TXNIP, whereas overexpression of miR-183 attenuated the neuropathic pain in CCI rats by inhibiting the TXNIP and NLRP3 expression. Recently, aberrant expression of miRNAs has been identified to affect the 


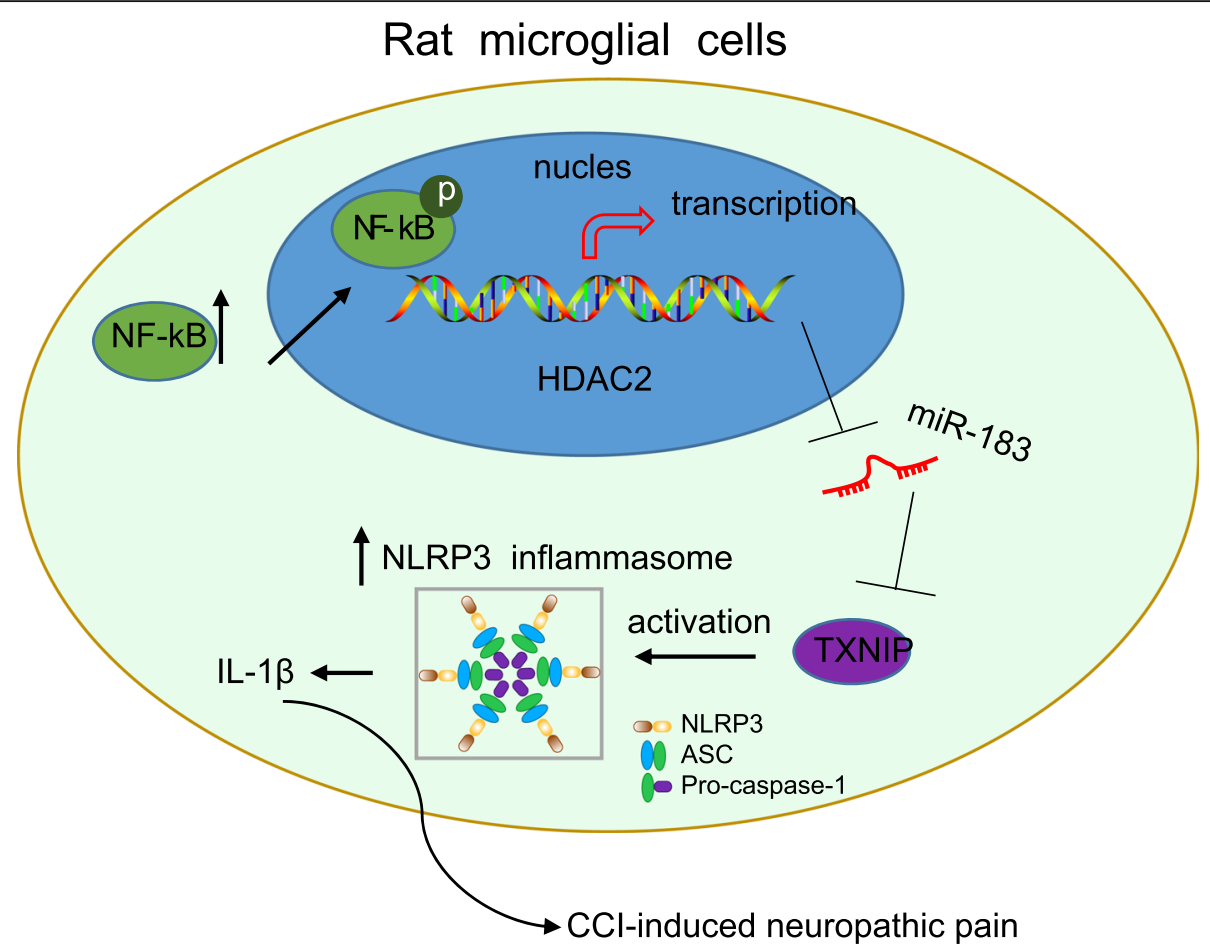

Fig. 7 Mechanism graph of NF-kB p65-mediated HDAC2 in CCl-induced neuropathic pain. NF-kB p65-activated HDAC2 could induce inflammation reaction to aggravate neuropathic pain in CCI rats by downregulating miR-183 and promoting TXNIP and NLRP3

development and progression of neuropathic pain [22]. On the other hand, miR-183 exhibits poor expression in neuropathic pain while restoration of miR-183 contributes to the attenuation of neuropathic pain [23]. Similarly, Shi et al. have illustrated the reduction of miR183-5p expression in dorsal root ganglion during the development of CCI-induced neuropathic pain, whereas the upregulation of miR-183-5p remarkably relieved neuropathic pain [12]. Nevertheless, TXNIP has emerged as a potential regulator of the formation of the NLRP3 inflammasome [13], whilst activation of NLRP3 inflammasome has reported contributing to the progression of neuropathic pain, indicating its ability to relieve neuropathic pain upon its inhibition [14]. Moreover, miR-23a has been documented to influence the development of neuropathic pain via regulating the TXNIP/ NLRP3 inflammasome axis in spinal glial cells [24]. Hence, we speculated that the upregulation of miR-183 could be attributed to great therapeutic potential in CCI-induced neuropathic pain by affecting inflammation response via inhibition of TXNIP and NLRP3.

\section{Conclusion}

In summary, the present study exhibited that NF- $\mathrm{KB}$ p65-activated HDAC2 could potentially induce inflammation reaction to aggravate neuropathic pain by downregulating miR-183 and promoting TXNIP and NLRP3 (Fig. 7). Thus, downregulated NF-kB p65-mediating
HDAC2 may function as a promising new direction for developing novel treatments for neuropathic pain. However, the research is still in the preclinical stage. Therefore, further investigations are required to unravel the underlying molecular mechanism.

\section{Abbreviations}

CCl: Chronic constriction injury; HDAC2: Histone deacetylase 2; NFKB: Nuclear factor-kappa B; TXNIP: Thioredoxininteracting protein; PDTC: Pyrrolidine dithiocarbamate; miR-183: MicroRNA-183; NLRP3: NLR family pyrin domain containing 3; ATCC: American Type Culture Collection; SD: Sprague-Dawley; oe: Overexpression; sh: Short hairpin; NC: Negative control; DMSO: Dimethyl sulfoxide; DMEM: Dulbecco's modified Eagle's medium; FBS: Fetal bovine serum; PWT: Paw withdrawal threshold; PWL: Paw withdrawal latency; WT: Wild type; MUT: Mutant; CDNA: Complementary DNA; RT-qPCR: Reverse transcription quantitative polymerase chain reaction; 3'UTR: 3'untranslated region; p: Phosphorylated; GAPDH: Glyceraldehyde-3phosphate dehydrogenase; IgG: Immunoglobulin G; BCA: Bicinchoninic acid; SDS-PAGE: Sodium dodecyl sulfate-polyacrylamide gel electrophoresis; PVDF: Polyvinylidene fluoride; ECL: Enhanced chemiluminescence; PBS: Phosphate-buffered saline; EDTA: Ethylenediaminetetraacetic acid; ELISA: Enzyme-linked immunosorbent assay; IOD: Integrated optical density; RIPA: Radioimmunoprecipitation assay; IL: Interleukin; OD: Optical density; CoIP: Co-immunoprecipitation; ChIP: Chromatin immunoprecipitation;

ANOVA: Analysis of variance

\section{Acknowledgements}

The authors would like to acknowledge the helpful comments on this paper received from the reviewers.

\section{Authors' contributions}

Jiamin Miao and Tianjiao Ji designed the study. Xuelong Zhou and Gang Chen collated the data, carried out data analyses, and produced the initial draft of the manuscript. Jiamin Miao and Tianjiao Ji contributed to drafting 
the manuscript. All authors have read and approved the final submitted manuscript.

\section{Funding}

This study was supported by the Natural Science Foundation of Zhejiang Province (No. LY16H090008).

\section{Availability of data and materials}

The datasets generated/analyzed during the current study are available.

\section{Ethics approval and consent to participate}

Animal experiments were approved by the Ethics Committees of Zhejiang University School of Medicine and conducted in strict adherence with the Guide for the Care and Use of Laboratory animals published by the US National Institutes of Health. All efforts were made to minimize the number and suffering of the included animals.

\section{Consent for publication}

Not applicable.

\section{Competing interests}

The authors declare no conflicts of interest.

\section{Author details}

'Department of Anesthesiology, Sir Run Run Shaw Hospital, Zhejiang University School of Medicine, No. 3, Qingchun East Road, Jianggan District, Hangzhou 310012, Zhejiang Province, China. ${ }^{2}$ Department of Anesthesiology, The First Affiliated Hospital, College of Medicine, Zhejiang University, Hangzhou 310003, China. ${ }^{3}$ Laboratory for Biomaterials and Drug Delivery, Department of Anesthesiology, Boston Children's Hospital, Harvard Medical School, Boston 02115, USA.

Received: 10 January 2020 Accepted: 15 July 2020

Published online: 28 July 2020

\section{References}

1. Zilliox LA. Neuropathic pain. Continuum (Minneap Minn). 2017;23:512-32.

2. Li J, Zhao PP, Hao T, Wang D, Wang Y, Zhu YZ, Wu YQ, Zhou CH. Urotensin II inhibitor eases neuropathic pain by suppressing the JNK/NF-kappaB pathway. J Endocrinol. 2017;232:165-74.

3. Colloca L, Ludman T, Bouhassira D, Baron R, Dickenson AH, Yarnitsky D, Freeman R, Truini A, Attal N, Finnerup NB, et al. Neuropathic pain. Nat Rev Dis Primers. 2017;3:17002.

4. Cao J, Wang JS, Ren XH, Zang WD. Spinal sample showing p-JNK and P38 associated with the pain signaling transduction of glial cell in neuropathic pain. Spinal Cord. 2015;53:92-7.

5. Liu HY, Li YX, Hao YJ, Wang HY, Dai XY, Sun T, Yu JQ. Effects of oxymatrine on the neuropathic pain induced by chronic constriction injury in mice. CNS Neurosci Ther. 2012;18:1030-2.

6. Popiolek-Barczyk K, Makuch W, Rojewska E, Pilat D, Mika J. Inhibition of intracellular signaling pathways NF-kappaB and MEK1/2 attenuates neuropathic pain development and enhances morphine analgesia. Pharmacol Rep. 2014;66:845-51.

7. Yin Q, Fan Q, Zhao Y, Cheng MY, Liu H, Li J, Lu FF, Jia JT, Cheng W, Yan CD. Spinal NF-kappaB and chemokine ligand 5 expression during spinal glial cell activation in a neuropathic pain model. PLoS One. 2015;10:e0115120.

8. Wagner T, Kiweler N, Wolff K, Knauer SK, Brandl A, Hemmerich P, Dannenberg JH, Heinzel T, Schneider G, Kramer OH. Sumoylation of HDAC2 promotes NF-kappaB-dependent gene expression. Oncotarget. 2015;6:712335.

9. Descalzi G, Ikegami D, Ushijima T, Nestler EJ, Zachariou V, Narita M. Epigenetic mechanisms of chronic pain. Trends Neurosci. 2015;38:237-46.

10. Maiaru M, Morgan OB, Tochiki KK, Hobbiger EJ, Rajani K, Overington DW, Geranton SM. Complex regulation of the regulator of synaptic plasticity histone deacetylase 2 in the rodent dorsal horn after peripheral injury. J Neurochem. 2016;138:222-32.

11. Lodrini M, Oehme I, Schroeder C, Milde T, Schier MC, Kopp-Schneider A Schulte JH, Fischer M, De Preter K, Pattyn F, et al. MYCN and HDAC2 cooperate to repress miR-183 signaling in neuroblastoma. Nucleic Acids Res. 2013;41:6018-33.
12. Shi DN, Yuan YT, Ye D, Kang LM, Wen J, Chen HP. MiR-183-5p alleviates chronic constriction injury-induced neuropathic pain Through Inhibition of TREK-1. Neurochem Res. 2018;43:1143-9.

13. Choe JY, Kim SK. Quercetin and ascorbic acid suppress fructose-induced NLRP3 Inflammasome Activation by Blocking Intracellular Shuttling of TXNIP in Human Macrophage Cell Lines. Inflammation. 2017;40:980-94.

14. Jia M, Wu C, Gao F, Xiang H, Sun N, Peng P, Li J, Yuan X, Li H, Meng X, et al. Activation of NLRP3 inflammasome in peripheral nerve contributes to paclitaxel-induced neuropathic pain. Mol Pain. 2017;13:1744806917719804.

15. Montilla A, Zabala A, Matute C, Domercq M. Functional and metabolic characterization of microglia culture in a defined medium. Front Cell Neurosci. 2020;14:22.

16. Zheng D, Sabbagh JJ, Blair LJ, Darling AL, Wen X, Dickey CA. MicroRNA-511 binds to FKBP5 mRNA, which encodes a chaperone protein, and regulates neuronal differentiation. J Biol Chem. 2016;291:17897-906.

17. Li Z, Guo Y, Ren X, Rong L, Huang M, Cao J, Zang W. HDAC2, but not $\mathrm{HDAC1}$, regulates $\mathrm{Kv} 1.2$ expression to mediate neuropathic pain in $\mathrm{CCl}$ rats. Neuroscience. 2019;408:339-48.

18. Chen Y, Chen X, Yu J, Xu X, Wei X, Gu X, Liu C, Zhang D, Xu Z. JAB1 is involved in neuropathic pain by regulating JNK and NF-kappaB activation after chronic constriction injury. Neurochem Res. 2016;41:1119-29.

19. Fu ES, Zhang YP, Sagen J, Candiotti KA, Morton PD, Liebl DJ, Bethea JR, Brambilla R. Transgenic inhibition of glial NF-kappa B reduces pain behavior and inflammation after peripheral nerve injury. Pain. 2010;148:509-18.

20. Ibi D, de la Fuente RM, Kezunovic N, Muguruza C, Saunders JM, Gaitonde SA, Moreno JL, ljaz MK, Santosh V, Kozlenkov A, et al. Antipsychotic-induced Hdac2 transcription via NF-kappaB leads to synaptic and cognitive side effects. Nat Neurosci. 2017;20:1247-59.

21. Matsushita Y, Araki K, Omotuyi O, Mukae T, Ueda H. HDAC inhibitors restore C-fibre sensitivity in experimental neuropathic pain model. Br J Pharmacol. 2013;170:991-8

22. Xie XJ, Ma LG, Xi K, Fan DM, Li JG, Zhang Q, Zhang W. Effects of microRNA223 on morphine analgesic tolerance by targeting NLRP3 in a rat model of neuropathic pain. Mol Pain. 2017;13:1744806917706582.

23. Peng C, Li L, Zhang MD, Bengtsson Gonzales C, Parisien M, Belfer I, Usoskin D, Abdo H, Furlan A, Haring M, et al. miR-183 cluster scales mechanical pain sensitivity by regulating basal and neuropathic pain genes. Science. 2017; 356:1168-71.

24. Pan Z, Shan Q, Gu P, Wang XM, Tai LW, Sun M, Luo X, Sun L, Cheung CW. miRNA-23a/CXCR4 regulates neuropathic pain via directly targeting TXNIP/ NLRP3 inflammasome axis. J Neuroinflammation. 2018;15:29.

\section{Publisher's Note}

Springer Nature remains neutral with regard to jurisdictional claims in published maps and institutional affiliations.

\section{Ready to submit your research? Choose BMC and benefit from:}

- fast, convenient online submission

- thorough peer review by experienced researchers in your field

- rapid publication on acceptance

- support for research data, including large and complex data types

- gold Open Access which fosters wider collaboration and increased citations

- maximum visibility for your research: over $100 \mathrm{M}$ website views per year

At $\mathrm{BMC}$, research is always in progress.

Learn more biomedcentral.com/submissions 